\title{
MULTIDISCIPLINARY TREATMENT OF A VOLUMINOUS PELVIC TUMOR WITH GLUTEAL DEVELOPMENT - CASE PRESENTATION
}

doi: 10.2478/rojost-2018-0017

\author{
E. Brătilă' ${ }^{1}$ C. Cârstoiu' ${ }^{2}$ D.E. Comandaşu \\ ${ }^{1}$ Department of Obstetrics and Gynecology, "Carol Davila" University of Medicine and Pharmacy, Bucharest, \\ Romania; "Prof. Dr. Panait Sârbu" Clinical Obstetrics and Gynecology Hospital, Bucharest, Romania \\ ${ }^{2}$ Department of Orthopedics and Traumatology, "Carol Davila" University of Medicine and Pharmacy, \\ Bucharest, Romania; University Emergency Hospital, Bucharest, Romania
}

The aim of the paper was to present the case of a 64 -year-old patient with no significant pathological history, accusing the significant increase in volume of her left buttock, accompanied by sciatic compression and pelvic pain phenomena. Clinical evaluation revealed a large, left gluteal tumor, of regular shape and medium consistency, painless at palpation, causing hyperesthesia in the sciatic territory by compression. The IRM with contrast enhancement described a heterogeneous tumor, measuring about 25 centimeters in diameter, isointense in the $T_{1}$ and $T_{2}$ signals, with hypointense areas and imagistic aspect suggesting differential diagnosis between liposarcoma and fibrolipoma. From the imagistic point of view, the origin of the tumor was pelvic, with a threadlike tract in the sciatic hole and development in the gluteal region for the most part.

Surgery was decided on a multidisciplinary team including a gynecologist, orthopaedist, and general surgeon, using a double approach through laparotomy and gluteal incision. The first operative step consisted in laparotomy with pelvic tumor dissection at the level of the iliac vessels and its prolongation at the level of the sciatic hole. The large gluteal incision was used during the second step in order to mobilize the tumor form between the gluteal fibers. Subsequent thorough hemostasis was performed with postoperative aspect inspection through both approaches. The postoperative evolution was favorable without immediate or late complications. The histopathological result was fibrolipoma, with good prognosis for the patient. In conclusion, the multidisciplinary surgical approach in this patient presenting with a pelvic tumor with transischial gluteal development was the optimal choice, which led to a favorable outcome in a complex case. Keywords: voluminous gluteal tumor, fibrolipoma, multidisciplinary team 